\title{
Inspecting Objects: Visibility Manoeuvres in Laparoscopic Surgery
}

\author{
Jeff Bezemer, Ged Murtagh and Alexandra Cope
}

\section{$1 \quad$ Introduction}

The successful accomplishment of a surgical procedure is contingent on visual access to the body parts on which the surgeons operate. Concern for visibility is evident in many of the practices surgeons employ, ranging from changing their positions and posture at the operating table to mobilising, cleaning up, and inspecting structures inside the patient's body. In this chapter we examine episodes where surgeons inspect areas of interest prior to making invasive manoeuvres that could damage vital structures in the patient's body. The aim of the chapter is to explore how these inspections are socially organized and practically accomplished by the surgeons.

The chapter approaches 'seeing' as an embodied activity. It takes as its starting point the notion of 'co-operative action' (Goodwin 2018), and builds on prior video-based studies on seeing in surgery (e.g. Koschmann et al. 2011, Koschmann and Zemel 2011, Mondada 2003, 2014, Cope et al. 2015). Much of this work has explored the ways in which members of a profession identify objects in the material environment, typically using pointing gestures, and classify them as instances of more general, named categories that make up their 'professional vision' (Goodwin 2018). The fragments we present come from video recordings of surgical operations in a teaching hospital, and illustrate this type of classificatory work: they feature surgeons who come to jointly see, name, and discuss objects inside the patient that are well described in the surgical-anatomical literature, yet that need to be newly recognized in each and every case 'for another next first time' (Garfinkel 1996).

The distinct contribution we aim to make in this chapter is to draw attention to a significant 'seen but unnoticed' (Garfinkel 1964), and hitherto undocumented, feature of surgical work, namely the use of 'visibility manoeuvres' designed to inspect objects that are treated as potential targets for invasive action. Drawing on a collection of video clips featuring different surgical teams, we show that these manoeuvres are 'transitive gestures' in that they are operations on a material environment that are treated by surgeons as meaningful signs, rendering visible and/or characterising areas of interest in the operative field and demonstrating a concern with and method of dealing with a widely publicised surgical safety issue. In so doing we show how surgeons' embodied orientation to objects and their 'technical' micro-operations can be systematically documented and accounted for in socialsemiotic terms.

The chapter continues with contextualization of the study in terms of the conditions for 'seeing' in the laparoscopic surgical environment; conceptualisation of gesture; and data, transcription, and analysis. Following that we present nine fragments from our video corpus, illustrating visibility manoeuvres (e.g. splitting, stretching, flipping, tracing) used to demonstrate specific features of anatomical objects targeted for invasive action. In the discussion we explore how the visibility manoeuvres convey meaning in relation to surrounding talk in the fragments. We conclude with reflections on the significance of the gestures in terms of patient safety.

\section{$2 \quad$ Seeing in laparoscopic surgery}


Seeing in surgery has traditionally been characterised by the 'huddle' (Kendon 1990), with parties to an activity organizing themselves to establish a shared visual focus of attention (see Figure 4.1a). In this arrangement, members' proximity to the operating table, and more specifically to the 'operative field' (an isolated area on the patient's body) is the main factor affecting their view. Given that space around the operating table is limited, this arrangement produces a degree of visual inequality and a set of practices to manage this. In this traditional huddle, a consultant, for example, may momentarily step aside to allow trainees to get a better view of the areas they are working on.

A

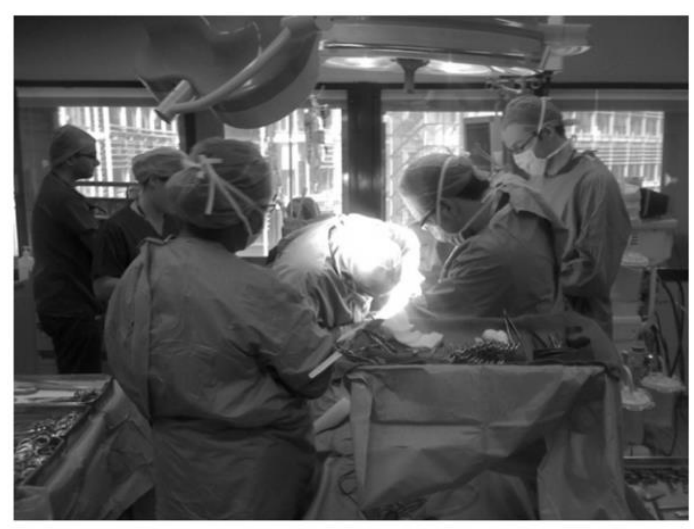

B

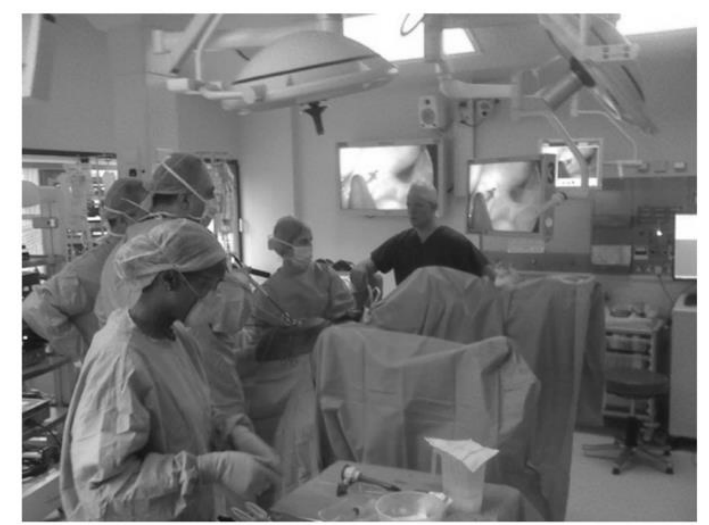

Figure 4.1: Open procedure (A) and laparoscopic procedure (B)

Laparoscopy has changed surgeons' visual access to the operative field. Instead of seeing areas directly, in laparoscopic ('keyhole', 'minimally invasive') surgery surgeons' view is mediated by the laparoscopic camera and monitors (see Figure 4.1b). This comes with visual gains (e.g. magnification) and losses (e.g. depth perception), and gives rise to new practices of seeing (e.g. camera control and its coordination with the operation; see Mondada 2014, Emerton-Coughlin et al. 2017). Crucially, the surgeons' bodily orientation shifts, as a video camera is used to obtain a view of the working space which is projected onto one or more video screens around the operating table. This arrangement creates a distributed yet shared point of reference for anyone participating: there is no need to be physically close to the patient to get a good view of the procedure (indeed a live feed of the laparoscope can give access from anywhere in the world). This arrangement makes the surgeon's work on the patient publicly visible and available for scrutiny and interpretation by anyone in the operating theatre with access to a monitor, of which there are now often several, as Figure $4.1 \mathrm{~b}$ illustrates. Thus, laparoscopy optimizes the possibilities for mutual monitoring (Goffman 1963) and puts operating surgeons on a 'stage', from where there is little possibility for hiding from those co-present, making surgeon's 'technical' actions on the patient also social actions.

The procedure we focus on in this chapter is to remove the gallbladder, a pear-shaped sac of bile that sits under the liver (see Figure 4.2). Before the gallbladder can be removed, two structures need to be identified, clipped, and cut: the cystic duct and the cystic artery (see Miranda 2016 for a very helpful diagram). In most patients, these structures are not visible when lifting the liver up as they are surrounded by fibrous and fatty tissue (Figure 4.3a). Only when this tissue is carefully removed do they become visible (Figure 4.3b). Thus visibility and certainty about the location of structures increases as dissection progresses (Hirschauer 1991, Prentice 2013). 


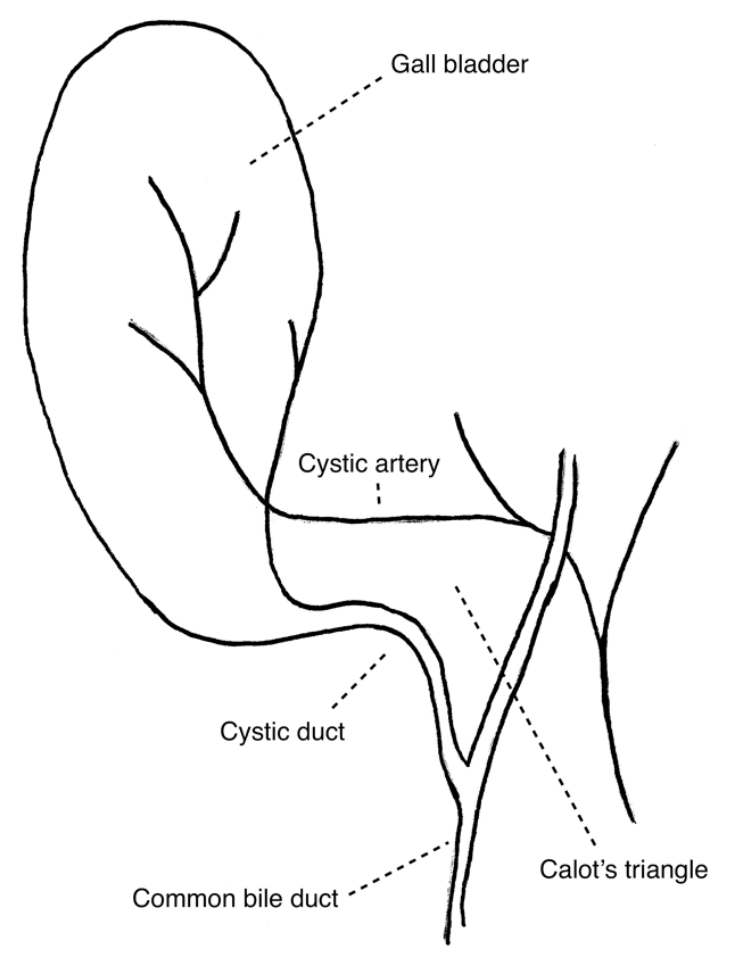

Figure 4.2: The Gall bladder

A

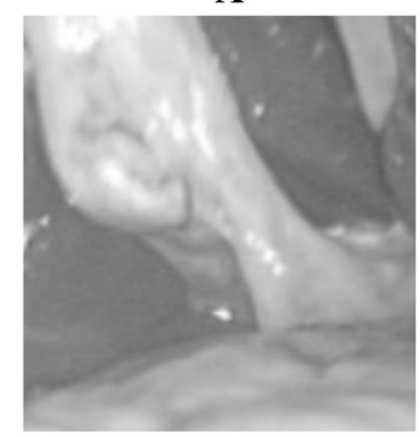

B

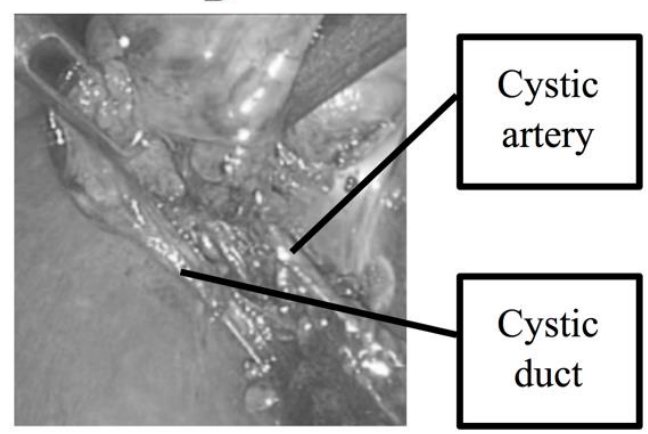

Figure 4.3: Cystic structures within 'Calot's triangle' before (A) and after (B) dissection

Dissection of the structures can take anywhere between several minutes to several hours. The completion of the dissection is a matter of clinical judgement. Soon after the introduction of laparoscopic cholecystectomy in the 1990s, reports were published showing a notable increase in cases where the cystic structures were misidentified, leading to injury of the common bile duct, which is considered a serious complication. Atul Gawande, an American surgeon-writer, puts it as follows:

Removing the gallbladder is fairly straightforward. ... There's one looming danger, though: the stalk of the gallbladder is a branch off the liver's only conduit for sending bile to the intestines for the digestion of fats. And if you accidentally injure this main bile duct, the bile backs up and starts to destroy the liver. Between 10 and 20 percent 
of the patients to whom this happens will die. Those who survive often have permanent liver damage and can go on to require liver transplantation. [...] It is a true surgical error, and, like any surgical team doing a lap chole, we were intent on avoiding this mistake. (Gawande 2003: 71)

One of the responses to the occurrence of misidentification has been the explication and teaching of identification 'methods'. One surgical textbook describes these methods as follows.

The cystic duct and artery must be carefully dissected and identified in the triangle of Calot to obtain the critical view. This critical view is achieved when the surgeon can see only two structures (the cystic duct and artery) entering directly into the gallbladder [...]; it must be obtained before any structures are clipped or transected. (Sherwinter 2018)

In another recent surgical publication, the 'Critical View of Safety' (CVS) is more broadly defined as 'demonstrating' that: (1) the lower part of the gallbladder is detached from the liver; (2) only two structures are attached to the gallbladder; and (3) these structures are freed from fatty and fibrous tissue (Strasberg et al. 2017). Gawande (2003), reflecting on a case he was involved in as a trainee, provides the following account:

Then, to be absolutely sure we were looking at the gallbladder duct and not the main bile duct, I stripped away some more of the surrounding tissue. The attending and I stopped at this point, as we always do, and discussed the anatomy. The neck of the gallbladder led straight into the tube we were eying. So it had to be the right duct. We had exposed a good length of it without a sign of the main bile duct. Everything looked perfect, we agreed. "Go for it," the attending said. (p. 72)

It is this moment of inspection, prior to dividing the cystic structures, that we explore in detail in this chapter.

\section{Gesture: A semiotic take on surgical manoeuvres}

As well as by talk, as Gawande suggests, the inspection is characterised by distinct, noninvasive manoeuvres. We treat these manoeuvres as gestures, semiotically defined as signs in which the 'signified' (a 'meaning') and the 'signifier' (a material 'form') have been brought together. Thus, we use the terms 'manoeuvre' or 'operation' to refer to the 'form' of a surgeon's operation on the patient's body, and we use the term 'gesture' to refer to the 'meaning potential' of these operations. The gesturer's choice of form is motivated, not arbitrary:

What that means is that the form is, in some of its aspects (characteristics, features) taken by the maker of the sign as being 'apt' to serve as the means of expressing the meaning at issue. In a ... laparoscopic operation, the surgeon makes a relatively restricted back and forth sideways movement with an instrument three times in quick succession. This gesture (the short back and forth movement with a 'grasper') 'means differently' to, say, a more extended movement, made more slowly, and made only once or twice. That is, the characteristics of this gesture are an apt means for what the 
surgeon wishes to communicate to his audience, namely 'a lack of obstruction'. (Bezemer and Kress 2016: 9)

Members of a surgical team often rely on the possibility that their visible bodily conduct is closely monitored by other members of the team. This enables the team members to coordinate their actions. Mondada (2011) provides an example of a surgeon holding an instrument that he uses to coagulate, and an assistant who operates the foot pedal that activates the coagulation. Her analysis shows that the assistant takes the surgeon approaching the tissue as a sign to activate the diathermy. The surgeon only used speech ("no co-ag") when he did not want the assistant to activate the diathermy as he was approaching tissue. Otherwise, he was expecting the assistant to treat his approaches as a request to activate the diathermy. This example defies the distinction often made between actions performed 'for the purposes of expression' and those made 'in the service of some practical aim' Kendon (2004: 15): the surgeon's 'approach' has the dual function of positioning the instrument and requesting activation of the diathermy.

The gestures we describe in this chapter are distinct in a number of ways. First, they are not part of a 'speech event' (Hymes 1962), but of what Goffman called a 'coordinated task activity' (1983), i.e. an activity that is organized around a practical task. In this type of activity, talk is intermittent, while gesture, as defined above, is almost continuous. Second, the gestures are operations on a material environment: they are 'transitive' gestures. These operations have physical and social effects; the act of manipulation and the outcome of that manipulation is monitored, evaluated, and interpreted. Third, we focus on operations made inside an enclosed space - the patient's abdomen - using long, thin instruments that are controlled from outside that space. The instruments serve as prosthetic extensions of the surgeon's body, mediating between two loci of action: the instrument handle (outside the patient's body) and the tip of the instrument (inside the patient's body). Laparoscopic surgeons, while operating on a patient, cannot touch the parts they are operating on directly with their bare hands. This means that the gestures used are different from the gestures commonly used in conversations (cf. Kendon 2004, Streeck 2008).

We explore the gestures in context. First, we attend to the semiotic relations between the operating surgeon's gestures and co-occurring talk. Goodwin notes that signs can be joined together to "create a whole that is both greater than and different from any of its constituent parts" (Goodwin 2000: 2). This is what Bezemer and Kress (2016) describe as 'sign complexes', that is, "a complex of coherent elements within coherent textual entities" (Bezemer and Kress 2016: 23). Second, we attend to the relations between 'sign complexes' produced at different points in time. This includes an interest in the ways in which a succession of sign complexes can project a range of likely next moves, such as the division of the cystic structures. Thus, we consider what gestures that are characteristic of surgeons' inspections of objects mean in the context of preceding and co-occurring signs.

\section{$4 \quad$ Materials and methods}

The study was conducted as part of a much larger study into surgery and surgical education at a major London teaching hospital. Within this larger study, audio and video data were captured with wireless microphones worn by at least one of the surgeons in each operation and with the laparoscopic camera used to capture the intra-corporeal instrument movements. All staff in the operating theatre and all patients involved gave informed consent. Participants were informed that the study was examining pedagogical practices in the operating theatre to provide insights that may be helpful to improve surgical training. Ethical approval was 
granted by the UK National Health Service Research Ethics Committee (Reference number 10/H0712/1).

The data presented derive from video recordings of 11 gallbladder operations. Each operation lasted between $20 \mathrm{~min}$ and $1 \mathrm{hr} 30 \mathrm{~min}$ (timing from the point that the laparoscope is inserted into the body cavity until the point it is taken out again). Total operating time across all 11 operations was $9 \mathrm{hr} 38 \mathrm{~min}$, with an average operating time of $52 \mathrm{~min}$. The surgical teams involved four different consultants (specialists, or, in U.S. terms, attendings); five specialty trainees (also called registrars, or, in U.S. terms, residents), ranging from 'ST3' to 'ST6', i.e. trainees who are in their third and sixth year of specialist training, respectively; one 'associate specialist' and one 'staff grade' (i.e. a non-training grade doctor); and a number of core trainees (i.e. junior surgeons on surgical rotations). In some cases, it was the consultant who operated, controlling the (metaphorical) scalpel, with assistance from one or more trainees, who controlled the laparoscopic camera and held a retractor in place to access the gallbladder. In other cases, it was the trainee who operated, with the consultant acting as camera person and/or supervisor.

All recordings were reviewed in order to produce first-pass transcripts of the spoken interaction; these transcripts were produced and reviewed by the authors, including one surgeon. We then proceeded with detailed transcription and examination of the transition from dissecting to dividing the cystic structures. This transition is marked by a distinct set of operations or manipulations on the patient's body. Unlike the type of operations performed before and after the inspection, these operations are non-invasive: they do not permanently change the patient. We described and compared these operations, first in isolation, and then in relation to their surrounding operations and talk. For prior analysis of the data, see Cope et al. (2015) and Bezemer et al. (2016).

Some notes on the transcripts we present are in order. First, they include descriptions of operations by the operating surgeon, relating to operations performed with their active hand; the other hand is normally in a more stable position as it is used to pull the gallbladder up. Stills are included to draw attention to selected features of operations at particular moments. These stills are sometimes fragments of the laparoscopic camera view; that is, they do not always show the whole picture that was available to the surgeons. Audio was transcribed in order to detail selected features of the talk, using the following conventions:

$\begin{array}{ll}\text { Cons } & \text { Consultant surgeon } \\ \text { STx } & \text { Surgical trainee (with x indicating year of specialty training) } \\ \text { SN } & \text { Scrub nurse } \\ (.) & \text { A full stop in brackets indicates a micro pause } \\ (1.0),(0.5) & \text { Silence in seconds and tenths of seconds } \\ . & \text { Falling, or final intonation contour } \\ ? & \text { Rising intonation } \\ , & \text { Continuing intonation }\end{array}$

The transcripts are broken down into numbered segments. Co-occurring speech and operations appear in the same numbered segment. For ease of reference we call these segments 'lines', even though they may include multiple lines.

Selected video fragments discussed in this chapter can be found at jeffbezemer.wordpress.com/video-clips.

\section{Visibility manoeuvres for demonstrating a window}


Figure 4.4 presents a plate of stills from three different cases, representing ways in which the surgeons use their instruments to demonstrate an opening or 'window' framed by structures on either side.

A

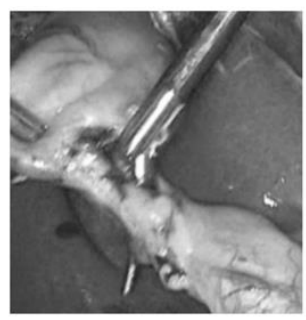

B

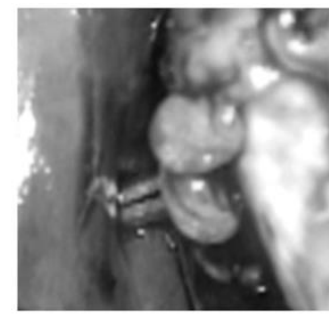

C

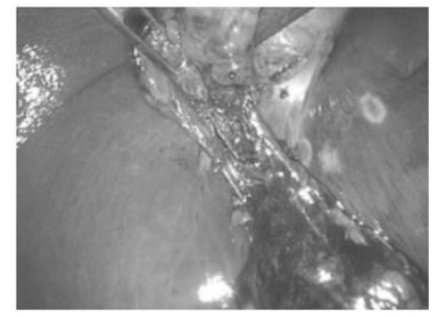

Figure 4.4: Demonstrations of windows

In Figure 4.4A, the surgeon's grasper has visibly come through an opening; his repeated 'splitting' of the grasper demonstrates the extent of the opening. In $4.4 \mathrm{~B}$, the surgeon has flipped the gallbladder over, and the tip of his closed grasper now visibly protrudes, thus demonstrating the opening. In $4.4 \mathrm{C}$, the surgeon has used his instrument to push the gallbladder up; this renders visible the window between the cystic duct and cystic artery, as well as the opening between the liver and the bottom part of the gallbladder. Note that in $4 \mathrm{~A}$ it is the movement that demonstrates an opening; in $4.4 \mathrm{~B}$ and $4.4 \mathrm{C}$, the instrument is held in a fixed position to demonstrate an opening. All demonstrations documented here are preceded and followed by invasive operations: they mark a transition from dissecting to dividing the structures being inspected.

Fragments 4.1-3 illustrate some of the different contexts within which these demonstrations were observed.

$1 \quad$ Cons Pushes a closed grasper through a thin layer of tissue, creating opening

2 Cons Makes series of small expansive movements when grasper has penetrated the tissue, creating a window

3 Cons Open the clips

$4 \quad$ Cons $\quad$ Places grasper inside window, opening and closing its blades

Line 4

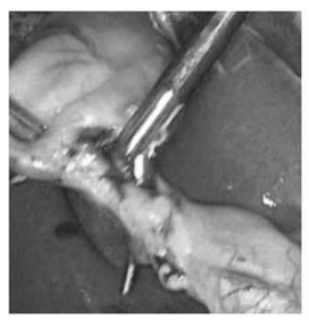

Fragment 4.1: Open the clips 
In Fragment 4.1, dissecting manoeuvres transition into visibility manoeuvres almost unnoticeably. In lines 1-2 the surgeon creates an opening by prodding in a thin layer of tissue with his instrument closed. When the tissue has separated, he starts widening the opening by opening the grasper out, as if making a split. Then, just seconds into the operation itself, he requests that the clip applicator be unpacked, thus signalling that he is planning to clip and divide a structure soon. The request provides the context for the subsequent 'splits', which can now be read as a demonstration of the evidence justifying this planned next move.

In Fragment 4.2, the consultant's running commentary turns the episode into a teaching episode: he 'translates' what he sees and does into generic nominal categories (window, critical view) and instructions for action (open up, line 1). He 'glosses' the critical view (when I ... know there's only one structure ... and nothing else, lines 4-7). At the same time, he also 'demonstrates' it: As he says that, he opens up that window (line 1) to obtain the critical view and ensures that there's only one structure (line 5). He then pushes a grasper through a window and exposes the other side of the gallbladder by flipping it over, thus showing that the tip of the instrument has come out the other end. He then flips the gallbladder back, and as he says and nothing else (line 7), he performs a splitting manoeuvre similar to that of the surgeon in Fragment 4.1, demonstrating the extent of the opening. This is followed by a request for the clip applicator, confirming that he is planning to progress to the next stage of the operation.

$\begin{array}{lll}1 & \text { Cons } & \text { And then I open up that window (.) oh no. Petelin again } \\ 2 & (2.0) & \\ 3 & \text { Cons } & \text { An I need to obtain what's called the critical view. } \\ 4 & \text { Cons } & \begin{array}{l}\text { Inserts closed grasper in opening } \\ \text { When I when I know }\end{array} \\ 5 & \text { Cons } & \begin{array}{l}\text { Flips gallbladder to other side } \\ \text { there's only one structure }\end{array} \\ 6 & \text { Cons } & \begin{array}{l}\text { Flips gallbladder back to position it was in before } \\ 7\end{array} \\ 8 & \text { Cons } & \begin{array}{l}\text { Opens grasper inside opening } \\ \text { and nothing else. }\end{array} \\ 8 & \text { Cons } & \text { Okay clip applicator }\end{array}$

Lines 1-3

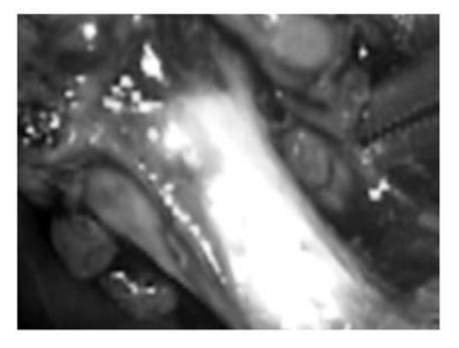

Line 5

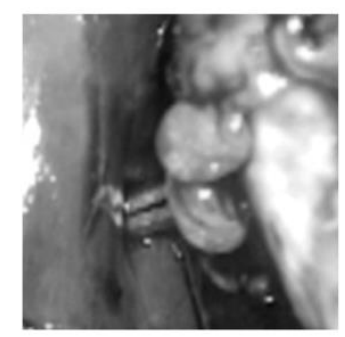

Line 7

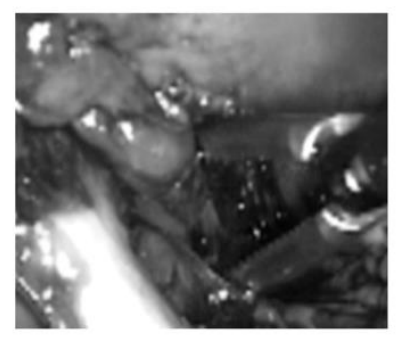

Fragment 4.2: I need to obtain what's called the critical view 
Fragment 4.3 shows how a window is demonstrated when a surgical trainee is operating under the supervision of a consultant. The trainee is working on the lower part of the structures shown in the still accompanying line 1, bluntly dissecting tiny adhesions by hooking them up.

\begin{tabular}{|c|c|c|}
\hline 1 & Cons & I think you're fine there now Michael. I'd jus I'd just take it all. \\
\hline 2 & ST4 & $\begin{array}{l}\text { Withdraws hook, pulls gallbladder down towards the liver } \\
\text { Clip em? }\end{array}$ \\
\hline & Cons & Yeah \\
\hline & ST4 & Pushes stuff down \\
\hline 5 & Cons & $\begin{array}{l}\text { because you can see if you just push up on the gallbladder, with your } \\
\text { hook just push up on the gallbladder go through there }\end{array}$ \\
\hline & ST4 & Abandons space, now placing hook under gallbladder and pushing it up \\
\hline & Cons & and you can see you've got a nice big window there. \\
\hline
\end{tabular}

Line 1

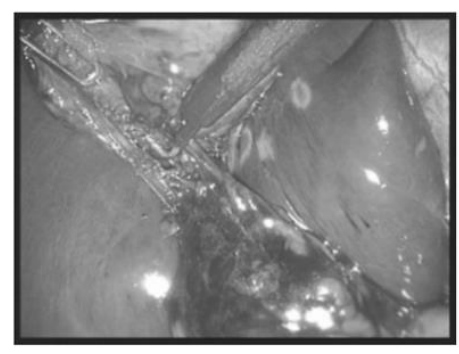

Line 4

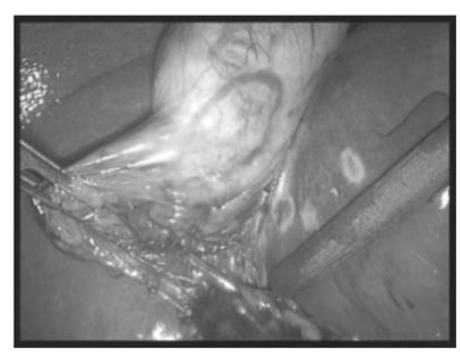

Line 6

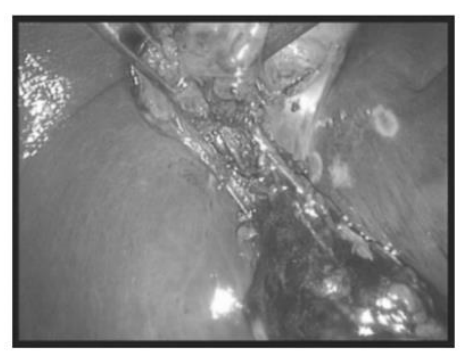

Fragment 4.3: I think you're fine there now

In line 1 the supervising consultant addresses the operator, offering his assessment of the state of the dissection (I think you're fine there now Michael) and proposes dividing the cystic structures (I'd just take it all). The trainee's response (clip em?, line 2) is given with rising intonation, thus taking the consultant's proposal as a directive that he is now asking the consultant to confirm. The consultant then confirms the trainee's version of his proposal. The proposal confirmation is followed by a 'justification' or reason for the proposed action (introduced by because, line 5), which is then provided, visually and verbally. To provide the visual justification, the operating trainee is instructed to 'remodel' the target structures. When the structures have been remodelled and fixed for 'intense scrutiny' (Goodwin 2018), the consultant draws attention to what you can now see: a nice big window there (line 7). Note that now the pronoun in you can see is used generically: It is not a claim by the consultant about what this trainee sees; rather it is a claim about what 'the surgical community' sees.

We found a similar pattern in Fragment 4.4, which is taken from another procedure where a trainee is operating. The consultant is scrubbed in and controls the camera. The cystic artery has already been divided. The trainee has just swapped his hook for a pledget, a type of 'wand'. 


\begin{tabular}{|c|c|c|}
\hline 1 & Cons & So this is what I call the critical view. \\
\hline 2 & ST5 & Yeah. \\
\hline 3 & Cons & Now push up on the gallbladder \\
\hline 4 & ST5 & Yeah yeah \\
\hline 5 & Cons & $\begin{array}{l}\text { Zooms camera in } \\
\text { Keep it there (.) push the gallbladder up }\end{array}$ \\
\hline 6 & Cons & You mean with the \\
\hline 7 & Cons & Go into the window (.) and push the gallbladder up \\
\hline 8 & Cons & $\begin{array}{l}\text { That's it. That's it. That's the view you } \\
\text { want. }\end{array}$ \\
\hline 9 & ST5 & Okay \\
\hline 10 & Cons & $\begin{array}{l}\text { That's the critical view. I'm happy for you to take the duct now if you } \\
\text { want. }\end{array}$ \\
\hline 11 & ST5 & Okay \\
\hline
\end{tabular}

Line 1

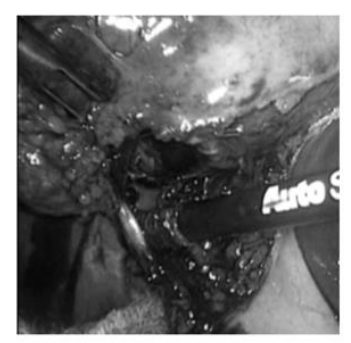

Line 8

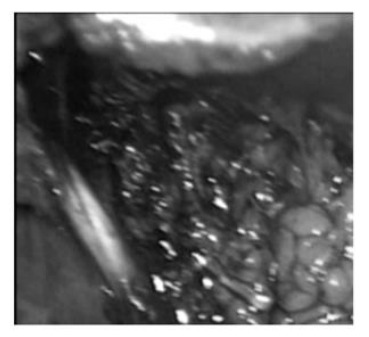

Fragment 4.4: That's what I call the critical view

In line 1 the consultant qualifies the state of the dissected structures: So this is what I call the critical view. When this is acknowledged by the operating trainee (line 2), the consultant instructs the trainee to remodel the structures. As he instructs the trainee on how to apply his instrument, the consultant, who controls the camera, zooms in on the area where the trainee is to push up on the gallbladder. Once the trainee has done so, the consultant confirms that that's the view you want (line 8) and that's the critical view (line 10). This qualification is immediately followed up by a proposal for the trainee to take the duct now if you want (line 10). Note that before the remodelling and fixing, and the zooming in on the area of interest, the consultant presented subjective qualifications (this is what I call...); once the conditions for visibility had been adjusted, he shifted to objective qualifications (that's the critical view).

\section{Visibility manoeuvres for demonstrating a cystic structure}


Figure 4.5 presents a plate of stills taken from five different cases (Figures $4.5 \mathrm{~B}$ and $4.5 \mathrm{~F}$ are from the same operation), illustrating the practice of 'tracing' the presumed cystic structures seconds before dividing them.

$\mathbf{A}$

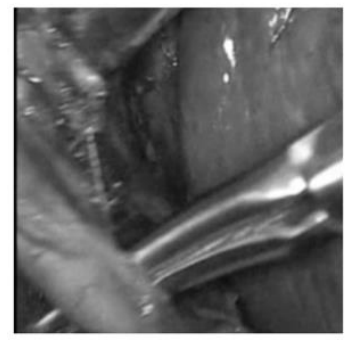

D

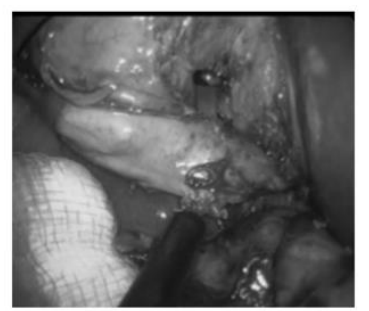

B

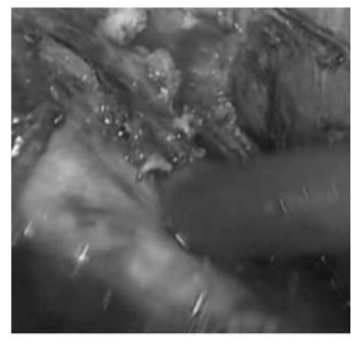

$\mathbf{E}$

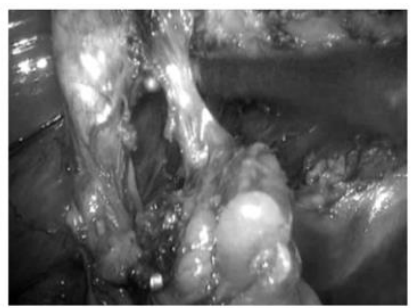

C

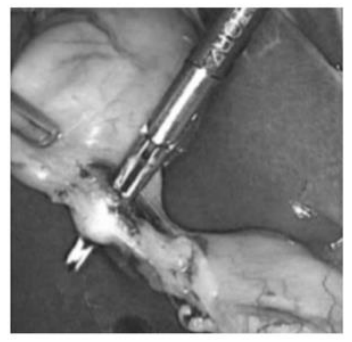

F

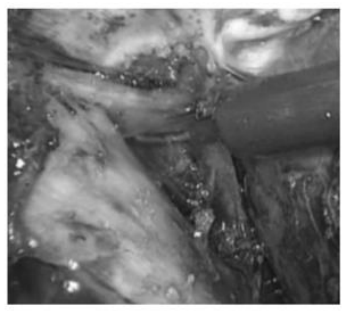

Figure 4.5: Demonstrations of cystic duct (A-D) and cystic artery (E-F)

All tracings documented here are preceded and followed by invasive operations: They mark a transition from dissecting to dividing the structures that are now being traced. They target (what we believe to be) the cystic duct (4.5A-D) and the cystic artery (4.5E-F), i.e. the structures that need to be divided.

The tracing is done with a curved grasper (4.5A and C), a hook (4.5B, E, and F), and a finger dissector (4.5D). These instruments allow the surgeon to 'hook up' the cystic structures as they move the instrument up and down. The tracing highlights a number of features. First, the radius of the trace renders visible the extent of the visible part of a structure. Second, the tracing shows the extent to which the structure is freed from surrounding tissue in areas to which the surgeon has limited visual access. For example, the trace in $4.5 \mathrm{D}$ shows that there is nothing behind the cystic duct. Third, the tracing has the potential to render visible gallstones inside the duct (which is relevant for its division) and pulsation in the artery (confirming it is an artery).

In $4.5 \mathrm{~B}$ and $4.5 \mathrm{~F}$ the duct and the artery are traced in quick succession, and from within the same opening. This 'pivoting' again highlights the opening or 'window' between the duct and the artery, which, unlike the window in $4.5 \mathrm{E}$ for example, is not visible unless it is stretched.

Fragments 4.5-7 illustrate some of the contexts in which these tracings were observed. 
1 Cons Drives hook into opening, tracing cystic duct

2 Cons Pushes cystic artery outward, exposing a window

I'm happy with that now.

3 Cons Traces and pushes cystic artery outward, exposing window

4 ST3 Yeah.

5 Cons Places hook inside window, moving it up and down, then pushes cystic artery outward, exposing window, then withdraws hook.

We'll try with clips otherwise we have to across with the

6 Cons Places hook in window, pushing cystic artery outward, exposing window, then withdraws hook.

$7 \quad$ Cons $\quad$ Okay clip applicator please

Line 1

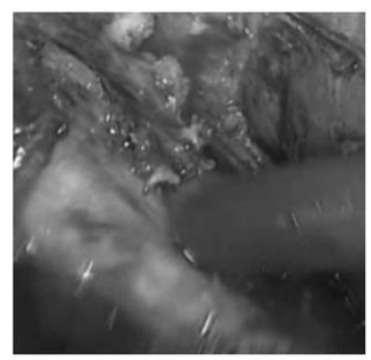

Line 3

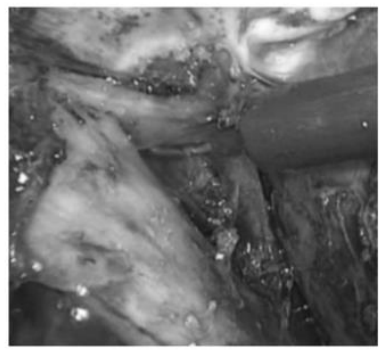

Fragment 4.5: I'm happy with that now

In this patient, the window that the surgeon has created is visible only when he manipulates it. The surgeon achieves this with the 'hook', the instrument previously used to dissect the area out. The consultant first traces the cystic duct. He then stretches the cystic artery out, while declaring that he is happy with that now (line 2), and traces it with the hook. The trainee acknowledges the declaration, and the consultant places the hook inside the window twice, moving it up and down against the cystic artery, stretching the structure outward, before requesting the clip applicator.

By declaring that he is happy with that now the consultant shares not so much his affective state as his assessment of the state of the structures he has just dissected (happy is frequently used in this way, as further fragments will show). His tracing of these structures during and immediately before and after the production of this phrase suggests that it is these structures that he is happy with. Thus the declaration retrospectively and prospectively frames the tracings as indexes of the objects he is happy with, and as demonstrations of evidence/support for his positive evaluation. Conjointly, the declaration and the tracing project and justify an invasive course of action, which is later confirmed by the consultant's request for the clip applicator.

One of the notable differences between an I need to obtain what's called the critical view (Fragment 4.2, line 3) and I'm happy with that now (Fragment 4.5, line 2) is that the former is framed pedagogically, 'naming' a common professional norm, while the latter statement refers to a personal professional norm, and does not name it. However, both 
surgeons use talk along with their tracings to mark the transition point between dissection and division, which is concluded in both cases with a request for the clip applicator.

In the following fragment a surgical trainee is operating 'independently', i.e. unsupervised, assisted by a slightly more junior trainee.

\begin{tabular}{|c|c|c|}
\hline 1 & ST6 & Prods thin layer of tissue with grasper, creating opening \\
\hline 2 & ST6 & Opens grasper, widening the opening just created \\
\hline 3 & ST6 & $\begin{array}{l}\text { Traces cystic duct } \\
\text { There you go }\end{array}$ \\
\hline 4 & ST5 & Nice \\
\hline 5 & ST6 & Agreed? \\
\hline 6 & ST6 & Places grasper closed behind cystic artery \\
\hline 7 & ST5 & $\begin{array}{l}\text { Zooms in } \\
\text { Yeah }\end{array}$ \\
\hline 8 & ST6 & Alex happy? \\
\hline 9 & ST6 & $\begin{array}{l}\text { Puts grasper behind cystic duct and flips gallbladder over and back } \\
\text { again }\end{array}$ \\
\hline 10 & Alex & Perfect. \\
\hline 11 & ST6 & Very good. \\
\hline
\end{tabular}

Line 3

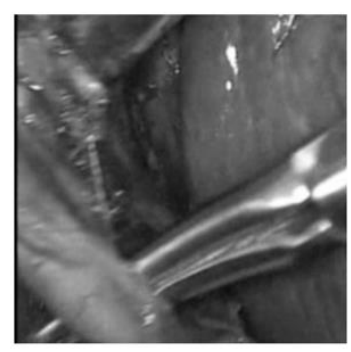

Line 6

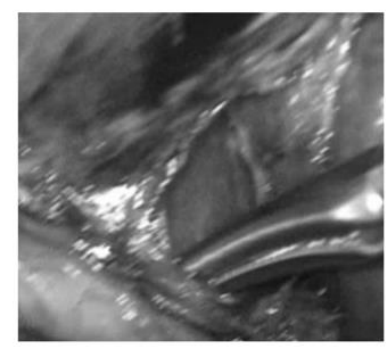

Line 9

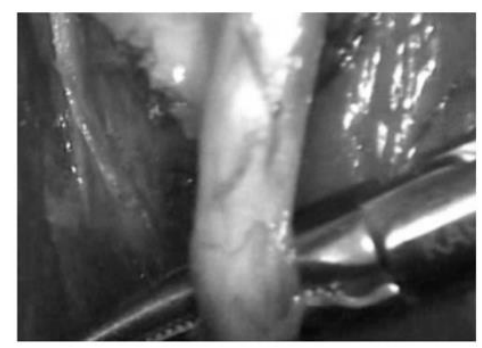

\section{Fragment 4.6: There you go}

The anatomy in this fragment is such that the cystic structures can be mobilised with a couple of dissecting manoeuvres. The operating surgeon then traces the duct, while claiming his accomplishment: there you go (line 3). The assistant responds with an aesthetic evaluation (nice, line 4), presumably of the anatomy, which is exceptionally clean and clear. The operator then seeks confirmation from his assistant and from Alex, the surgeon-researcher copresent (and co-author of this chapter). As he seeks their agreement, he places the instrument behind the cystic artery, pushing this structure sideways, and then places it behind the duct, flipping the gallbladder.

Fragment 4.7 continues from Fragment 4.1, and is from another 'easy anatomy' case. 


$\begin{array}{lll}5 & \text { Cons } & \text { Is the hook diathermy ready } \\ 6 & \text { SN } & \text { Yes. } \\ 7 & \text { Cons } & \begin{array}{l}\text { Places grasper inside window, opening and closing its blades twice and } \\ \text { tracing frame of window }\end{array} \\ 8 & \text { Cons } & \text { Clips } \\ 9 & (5.0) & \\ 10 & \text { Cons } & \text { This is gonna be over in a minute. } \uparrow \text { Clips } \\ 11 & \text { SN } & \text { Passes clip applicator } \\ 12 & \text { Cons } & \text { Thanks. }\end{array}$

Line 7

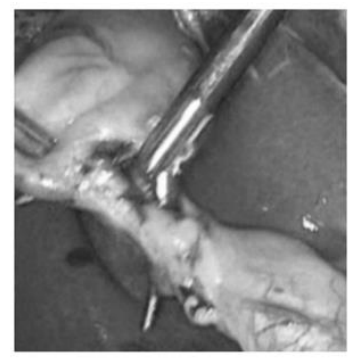

Line 7

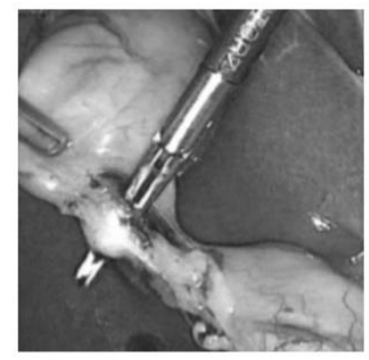

Fragment 4.7: Open the clips (cont.)

In this fragment tracings happen twice, and in both cases they are preceded by instrument requests directed at the scrub nurse. In line 3, the consultant asks the scrub nurse to open the clips, i.e. to unpack the boxed clip applicator, suggesting that he is planning to clip. In line 5, the consultant plans further ahead, asking the scrub nurse whether the hook diathermy is ready, i.e. the instrument he uses to dissect the gallbladder from the liver bed once the cystic structures have been divided. In line 8, he asks for the clip applicator. All the while, the consultant has traced structures, suggesting a target for and demonstrating the appropriateness of clipping. As in the previous fragments, the consultant's operations, along with his verbal requests for instruments, project a course of action, with the 'verbal' instrument requests indexing the type and timing of action, and the 'tracings' the target of the action. Note, however, that he does not use talk to verbally explicate his judgement and/or provide opportunities for his trainee to comment, as the surgeons in Fragments 4.2-6 did.

\section{$7 \quad$ Developing the justification to divide the cystic structures}

In some cases, the justification of the division continued after the request for the clip applicator. Fragments 4.8-9 illustrate this type of situation.

Fragment 4.8 continues from Fragment 4.3. 


\begin{tabular}{|c|c|c|}
\hline 8 & ST4 & Flips gallbladder over to expose other side \\
\hline 9 & Cons & $\begin{array}{l}\text { an I mean the ideal is you take all that stuff and you make a big window } \\
\text { but }\end{array}$ \\
\hline 10 & ST4 & Gently applies hook around cystic duct \\
\hline 11 & Cons & Flips gallbladder back and places hook under gallbladder \\
\hline 12 & ST4 & Do you think I'm gonna have to take it from this side? \\
\hline 13 & Cons & Yeah we::1l I think you're fine. \\
\hline 14 & ST4 & Hooks up tissue, applies diathermy \\
\hline 15 & Cons & There's something in there isn't there. \\
\hline 16 & ST4 & Lets go of tissue, hooks up cystic artery and traces it \\
\hline 17 & Cons & So you need to stay high on the gallbladder. So I'd take these structures. \\
\hline 18 & ST4 & Okay clips \\
\hline 19 & Cons & Take them around the gallbladder and you'll be fine. \\
\hline 20 & ST4 & Clips please \\
\hline 21 & $(7.0)$ & \\
\hline 22 & Cons & $\begin{array}{l}\text { So you got cystic duct cystic artery the whole thing is I mean this is a } \\
\text { fairly straightforward one but if you get a (2.0) difficult one you just } \\
\text { need to make all this (.) window so that's Calot's triangle so this is a } \\
\text { classic anatomy the artery going through (.) the middle of Calot's } \\
\text { triangle }\end{array}$ \\
\hline
\end{tabular}

Line 12

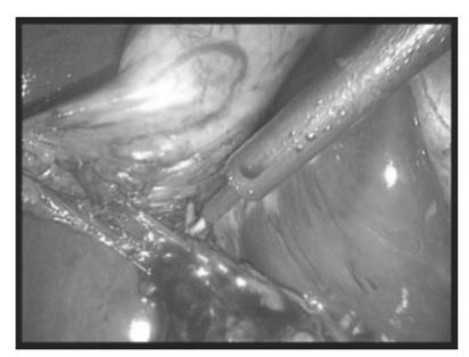

Line 14

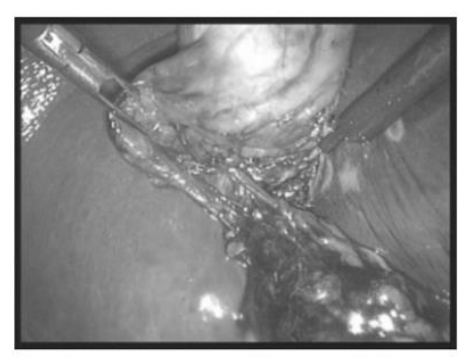

Line 16

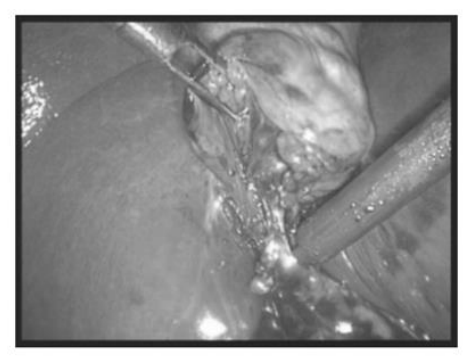

Fragment 4.8: I think you're fine there now (cont.)

Prompted by the consultant, the trainee in line 7 of Fragment 4.3 (just before Fragment 4.8 begins) exhibits a nice big window there, which is viewed from different angles by pushing up on the gallbladder and flipping it over to the other side. In the following lines, the consultant continues to prompt the trainee to proceed to divide the cystic structures without further delay and provides instructions on where to place the clips. This is concluded in line 20, when the trainee requests the clip applicator. 
As the trainee is waiting to receive the clip applicator, the consultant starts an 'elaboration' (line 22). He (re)states that they have identified the cystic duct and the cystic artery (so you got cystic duct cystic artery). Then he compares the present (straightforward) case to other cases ( $a$ difficult one), and similarly to Fragment 4.2 produces an online commentary, again providing the background schema for how to competently perform the procedure, and moving beyond the specifics of the case.

The surgeon's comments serve to justify his proposal to take these structures (line 17) and not to dissect any further, in spite of not actually having achieved the 'ideal' yet. At the same time, they are a way of orienting to the trainee's needs by formulating more general principles and rules of thumb that can be applied in future cases.

Note that in this fragment the notion of the critical view is invoked without using the term. The term was used, however, in the case from which Fragment 4.9 is taken. Fragment 4.9 starts at the point where the operating consultant has received the clip applicator. They have already discussed 'the critical view' at length, and the consultant has emphasized that it's all about safety (before the fragment begins).

1 Cons Drives in clip applicator

Please that's what I want in all the cases. Is that okay?

2 ST5 Yeah yeah it's more than okay.

3 Cons Touches cystic artery, then traces cystic duct with clip applicator Here is definitely going into the gallbladder and is not continuous, continuous with the $\mathrm{x}$ or the cystic duct

$4 \quad$ Cons $\quad$ Puts one clip in

5 Cons camera b down

$6 \quad$ ST5 Changes camera position

7 Cons You see? Miles away now from (.) here's the common bile duct, miles away yeah?

Line 3

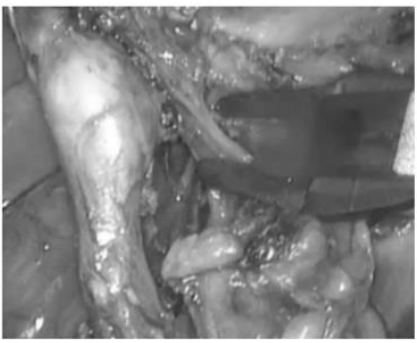

Line 3

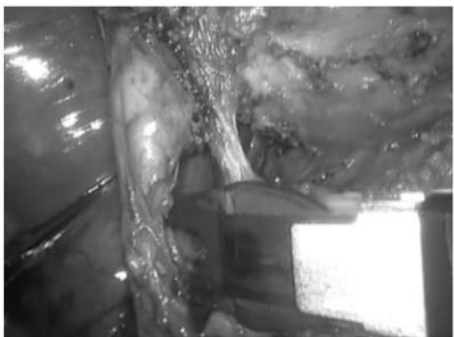

Line 7

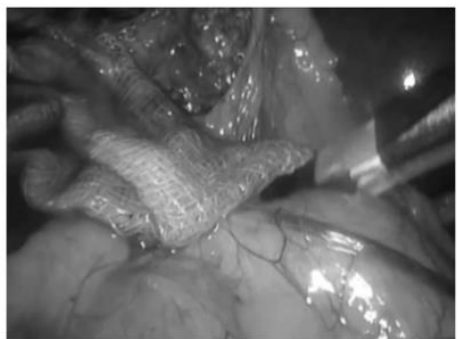

Fragment 4.9: That's what I want in all cases

As the operating consultant puts clips in the artery, he turns the view in that moment into a model for future action by the trainee, who is asked to confirm that he accepts this model for future operations that he might perform independently on this consultant's patients. 
The format of this request (line 1) leaves little room for disagreement, and the trainee hastens to commit to the consultant's proposed standard (line 2).

A little later, when the consultant has driven the clip applicator in, he draws attention to more evidence that the critical view has been obtained. First he points to and traces (with the clip applicator) the (top) area where the presumed cystic duct definitely (line 3) enters the gallbladder, and second to the (bottom) area, miles away (line 7) from the area they were just focusing on, where the consultant believes the cystic duct joins the common bile duct (this is covered in fat). The latter requires the trainee to change the camera position.

This is another example of an elaboration, which again is pedagogically framed, with attention drawn by the consultant to the target structures and the non-target structures (the common bile duct), and the view set as a standard for future cases.

\section{Discussion}

Taken together, the fragments illustrate different ways in which inspections prior to the division of the cystic structures are accomplished. Characteristic of all inspections is the use of visibility manoeuvres, chief among them the tracing of structures. So what meanings do these tracings convey and how? If they function as gestures, how might we characterize the relation between signifier and signified?

In a social semiotic account (Kress 2010, Bezemer and Kress 2016), the tracings are approached in the same way as any other mode of representation, first, in that they are partial: They bring out 'selected' features of the object being represented, namely those considered criterial by the sign maker. The materials we have presented show that to the experienced surgeons at our research site, criterial features of the cystic structures include shape, size, and 'freeness' (from their surrounding elements). Second, as in all meaning making, the sign maker draws on the distinct possibilities of the semiotic resources available: here, the resources of surgical instruments and manual operations. The relation between signifier and signified is motivated, not arbitrary: The virtual lines drawn through tracing resemble (like an icon) the shape and size of the structure that the tracing is representing.

Yet, the meaning potential of the tracings is not bounded by their contiguity with the material environment. Indeed, the tracings point in two directions. On the one hand, they represent (selected features of) unique structures in an actual patient; on the other, they stand for a 'token' of an abstract surgical 'type' or category. Outsiders may recognize (some of) the former, but only insiders will recognize the latter. To outsiders, the tracing might draw attention to some of the features that the surgeon wishes to highlight; that is, they might couple the tracing with referents in the here-and-now. To an insider, the tracing is also coupled with (candidate) referents beyond the here-and-now, e.g. with features highlighted in diagrammatical, idealized representations of the structure such as those provided in surgical textbooks.

Thus, notwithstanding the non-arbitrary relation between signifier and signified, the tracings are far from 'self-explanatory'. They are part of a shared set of gestures that every new surgical trainee develops, along with the surgical-anatomical lexicon, and as such they are instrumental in trainees' learning what and how to see. This constitutes an important footnote to Polanyi's oft-quoted discussion of medical students learning to read X-rays, who initially "can see nothing that [expert staff] are talking about" (1958: 101): Tracings and other gestures (radiologists will have developed a set of their own) are essential links between seeing and talking.

The gestures we described were surrounded by talk. Prior work on the relation between gesture and speech has shown that pointing gestures often help identify in the 
material environment the referent of deictic elements in co-occurring speech. Sometimes these gestures fill the grammatical position of those elements, as in one of Goodwin's (2018) archaeologists, who said "Wha'do you think of $u$ ", as she was pointing at an area in the soil. In these well-documented cases, speech and gesture are usually strictly temporally anchored: In Goodwin's example, the pointing coincides with and remains fixed throughout the spoken utterance.

Our materials reveal a differently configured multimodal sign complex. We have shown that verbal deixis often occurs in the midst of a series of tracings in different places. For instance, in Fragment 4.5, the operating surgeon's I'm happy with that now (line 2) coincides with but is also directly preceded and followed by tracings, of both the cystic duct and the cystic artery. All of these tracings identify candidate referents of that. In Fragment 4.6, the operating surgeon seeks confirmation from his colleagues by asking agreed? (line 5) and happy? (line 8 ). While the operator's proposals do not contain any deixis pointing to elements in the material environment, they can only be understood in relation to that environment and the series of gestures made in it, which identify a range of candidate complements of the proposal, specifying what the other person is asked to agree on and/or be happy with. Thus in these examples, a single spoken utterance is coupled with a series of gestures produced in a partially overlapping time frame.

\section{Conclusion}

We have presented and discussed the surgical inspection of anatomical objects in laparoscopic gallbladder removal procedures in terms of instrument-enhanced, non-invasive visibility manoeuvres. We explored, first, manoeuvres designed to demonstrate a window (splitting, stretching, flipping); and second, manoeuvres designed to demonstrate features of anatomical structures (tracing). We presented video fragments from a range of different cases, including some with consultants acting as operators and others with trainees acting as operators.

To highlight their semiotic potential, we described the manoeuvres as 'transitive gestures', showing that they render visible defining features of particular objects of interest, which are only sometimes made explicit in surrounding talk. The gestures were always performed just before the actual clipping of the cystic structures. Yet they do not by themselves project the clipping of these structures. It is through the gestures' combinations with surrounding actions, such as a verbal request for a clip applicator, a verbal evaluation, and/or a verbal proposal for the next action, that sign complexes pointing to the next action are formed. Within these complexes, the gestures carry distinct meanings, identifying the target objects of the next action, and justifying that action by rendering visible criterial features of the target objects. Above all, the gestures demonstrate that operators intensely scrutinise the objects they are planning to invade. Thus they are a means of displaying an orientation to the mitigation of risk, and of acknowledging a widely promoted surgical safety procedure, in the absence of, or alongside, talk that explicitly or implicitly acknowledges that risk.

By offering an account of the meaning potential of commonly used practical actions, we have drawn attention to significant 'seen but unnoticed' (Garfinkel 1964), and hitherto undocumented, features of embodied activity in surgery. We have shown how visibility manoeuvres are both technical and social actions, and how a concern for a specific, welldocumented risk to the safety of the patient translates, at a micro-level, into practices of seeing. That, we believe, is precisely what can be gained from perspectives that recognize the role of the body in situated social interaction and the material environment it is acting in and 
on. They invite us to notice, document, and analyse how all bodily ('technical') operations, not just the movement of speech articulators, have expressive potential, are part of larger semiotic complexes, and are treated as such by those noticing them, not least in settings where lives are at stake.

\section{References}

Bezemer, Jeff and Gunther Kress. 2016. Multimodality, learning and communication: A social semiotic frame. London: Routledge.

Bezemer, Jeff, Ged Murtagh, Alexandra Cope, and Roger Kneebone. 2016. Surgical decision making in a teaching hospital: A linguistic analysis. ANZ Journal of Surgery 86(10): 751-755.

Cope, Alexandra, Jeff Bezemer, Roger Kneebone, and Lorelei Lingard. 2015. You see? Teaching and learning how to interpret visual cues during surgery. Medical Education 49(11): 1103-1116.

Emmerton-Coughlin, Heather, Christopher Schlachta, and Lorelei Lingard. 2017. 'The other right': Control strategies and the role of language use in laparoscopic training. Medical Education 51(12): 1269-1276.

Garfinkel, Harold. 1964. Studies of the routine grounds of everyday activities. Social Problems 11(3): 225-250.

Garfinkel, Harold. 1996. Ethnomethodology's program. Social Psychology Quarterly 9(1): 5-21.

Gawande, Atul. 2002. Complications: A surgeon's notes on an imperfect science. London: Profile Books.

Goffman, Erving. 1963. Stigma: Notes on the management of spoiled identity. London: Penguin.

Goffman, Erving. 1983. The interaction order: American Sociological Association, 1982 Presidential Address. American Sociological Review 48(1): 1-17.

Goodwin, Charles. 2000. Action and embodiment within situated human interaction. Journal of Pragmatics 32(10): 1489-1522.

Goodwin, Charles. 2018. Co-operative action. Cambridge: Cambridge University Press.

Hirschauer, Stefan. 1991. The manufacture of bodies in surgery. Social Studies of Science 21(2): 279-319.

Hymes, Dell. 1962. The ethnography of speaking. In Anthropology and human behavior, eds. Thomas Gladwin and William C. Sturtevant, 13-53. Washington: Anthropological Society Washington.

Kendon, Adam. 1990. Conducting interaction: Patterns of behavior in focused encounters. Cambridge: Cambridge University Press.

Kendon, Adam. 2004. Gesture: Visible action as utterance. Cambridge: Cambridge University Press.

Koschmann, Timothy, Curtis LeBaron, Charles Goodwin, and Paul Feltovich. 2011. "Can you see the cystic artery yet?": A simple matter of trust. Journal of Pragmatics 43(2): 521-541.

Koschmann Timothy and Alan Zemel. 2011. "So that's the ureter.": The informal logic of discovering work. Ethnographic Studies 12: 31-46.

Miranda, Efrain A. 2016. Medical terminology daily. https://www.clinanat.com/mtd/739triangle-of-calot. 
Mondada, Lorenza. 2003. Working with video: How surgeons produce video records of their actions. Visual Studies 18: 58-73.

Mondada, Lorenza. 2014. The surgeon as a camera director: Maneuvering video in the operating theatre. In Studies of video practices: Video at work, eds. Mathias Broth, Eric Laurier, and Lorenza Mondada, 97-132. Abingdon, UK: Routledge.

Polanyi, Michael. 1958. Personal knowledge: Towards a post-critical philosophy. Chicago: University of Chicago Press.

Prentice, Rachel. 2012. Bodies in formation: An ethnography of anatomy and surgery education. Durham: Duke University Press.

Strasberg, Steven M. and L. Michael Brunt. 2017. The critical view of safety: Why it is not the only method of ductal identification within the standard of care in laparoscopic cholecystectomy. Annals of Surgery 265(3): 464-465.

Streeck, Jürgen. 2008. Depicting by gesture. Gesture 8(3): 285-301. 